Research Article

\title{
Transmission Dynamics of Resistant Bacteria in a Predator-Prey System
}

\author{
Xubin Gao,, ${ }^{1,2}$ Qiuhui Pan, ${ }^{1,3}$ and Mingfeng $\mathrm{He}^{1}$ \\ ${ }^{1}$ School of Mathematical Sciences, Dalian University of Technology, Dalian 116024, China \\ ${ }^{2}$ City institute, Dalian University of Technology, Dalian 116600, China \\ ${ }^{3}$ School of Innovation Experiment, Dalian University of Technology, Dalian 116024, China \\ Correspondence should be addressed to Mingfeng He; mfhe@dlut.edu.cn
}

Received 8 October 2014; Revised 7 January 2015; Accepted 7 January 2015

Academic Editor: Maria N. D. S. Cordeiro

Copyright (C) 2015 Xubin Gao et al. This is an open access article distributed under the Creative Commons Attribution License, which permits unrestricted use, distribution, and reproduction in any medium, provided the original work is properly cited.

\begin{abstract}
This paper discusses the impact on human health caused by the addition of antibiotics in the feed of food animals. We use the established transmission rule of resistant bacteria and combine it with a predator-prey system to determine a differential equations model. The equations have three steady equilibrium points corresponding to three population dynamics states under the influence of resistant bacteria. In order to quantitatively analyze the stability of the equilibrium points, we focused on the basic reproduction numbers. Then, both the local and global stability of the equilibrium points were quantitatively analyzed by using essential mathematical methods. Numerical results are provided to relate our model properties to some interesting biological cases. Finally, we discuss the effect of the two main parameters of the model, the proportion of antibiotics added to feed and the predation rate, and estimate the human health impacts related to the amount of feed antibiotics used. We further propose an approach for the prevention of the large-scale spread of resistant bacteria and illustrate the necessity of controlling the amount of in-feed antibiotics used.
\end{abstract}

\section{Introduction}

As the world's population continues to increase, methods for improving the production of livestock have become a great challenge. The growth-promoting effects of antibiotics were first discovered in the 1940s when chickens fed by-products of tetracycline fermentation were found to grow faster than those not fed with these by-products [1]. Since then, many antimicrobials have been found to improve average daily weight gain and feed efficiency in livestock in a variety of applications [2-4], and this process has come to be known as "growth promotion." Antibiotics used in feeding not only increase production but can also prevent disease. Infected animals will not only affect the production capacity, but as the stocking density increases, infection is more likely to be transmitted at a faster rate. Therefore, the most effective strategy to prevent epidemics in livestock is to add antibiotics to the animal feed, which has become common practice [5].
Although the use of antibiotics as feed additives can promote animal growth, there are also various side effects that affect the human consumer. When antibiotics are used in animal feed, resistance is likely to be selected for in the normal and infected bodies, and therefore increase in prevalence in the population [6-10]. Furthermore, Perreten et al. [11] demonstrated that resistant bacteria are highly contagious, and resistance can transfer to other strains through plasmids or by direct contact. Therefore, other congeneric animals can readily and rapidly become infected with antibiotic-resistant bacteria.

Resistant bacteria not only transmit horizontally but can also transmit across species. When animals harboring antibiotic-resistant bacteria come in close contact with humans or are consumed by humans, the resistant bacteria may transfer to the humans [12].

Harwood et al. [13] confirmed that resistant bacteria could spread through sewage to reach the general 
environment. Consequently, if birds or other types of urban wildlife were to drink the sewage, the resistant bacteria would spread widely either through their feces or if directly consumed by other animals. Resistant bacteria have been found in wild rodents and in some domestic animals [14, 15]. Direct contact is another important way that resistant bacteria can transfer from animals to humans. Van den Bogaard et al. [16] and Donabedian et al. [17] conducted experiments that demonstrated that resistant bacteria do indeed have the ability of being transmitted from animals to humans. Anderson et al. [18] indicated that different types of resistant bacteria present in the food chain have different effects on humans. In a review, Phillips et al. [19] explained that the use of antibiotics in food animals poses a risk to human health and listed the various ways that resistant bacteria can transfer from animals to humans through the food chain and by other means. Tollefson and Miller [20] described the history of antimicrobial use and regulation in animals, the related public health concern, the current animal drug approval process in the United States, the international perspective, and the Food and Drug Administration's (FDA's) proposed procedures for evaluating the human health impact of the antimicrobial effects associated with animal drugs intended for use in food-producing animals. All of the articles cited above clearly show that resistant bacteria can indeed transfer from food animals to humans through direct contact or consumption.

Once humans are infected with resistant bacteria, the bacteria will readily transfer among the human population. In order to prevent the wide spread of resistant bacteria among humans, many researchers have studied the spread process of resistant bacteria between humans in the hospital and have proposed prevention measures [21-29]. However, almost no quantitative analysis of the spread of resistant bacteria from animals to humans has been performed to date. In particular, no study has yet focused on the process of transmission through the food chain, in which food animals consume large amounts of antibiotic resistant bacteria and then transmit the resistant bacteria to humans through direct contact or predation, and the bacteria continue to spread among humans. In this paper, we discuss this process and report the results of a quantitative analysis of the effects that the amount of antibiotics used as feed additive have for human health.

Based on the resistant bacteria transmission rule, we use Lotka-Volterra equations [30] to derive a model of the spread of resistant bacteria in a predator-prey system. Resistant bacteria can be viewed as an infectious disease in a predator-prey system [31-36]. The predator-prey systems are key factors in ecological systems. So many articles have focused on the spread of infectious diseases in a predator-prey system [3743]. Some researchers have adopted a predator-prey model with only the prey affected by disease and some of them are representative: Anderson and May [44], Chattopadhyay et al. [35, 43], Xiao and Chen [45], Greenhalgh and Haque [46], and Sinha et al. [47-52], whereas others have modeled disease in only the predator, for instants, Haque and Venturino [53, 54], Hilker and Schmitz [55], and Auger et al. [56]. Few studies consider the predator-prey models with disease in both predator and prey. Hsieh and Hsiao [57], Pada Das et al. [58], and Gao et al. [59] modeled the situation in which both the predator and the prey have the disease, but in the first article, the disease was not contagious among the predators, and in the second article, there was no cross-infection, and in the third article, the diseases were transmitted vertically in both populations. Briggs and Hoopes [60], Chaudhuri et al. $[61,62]$ discussed cross-infection model. Furthermore, two other models $[63,64]$ were presented in which the disease could cross the species barrier in an interacting population. We here present a model in which the infected disease is represented by resistant bacteria that can be transmitted between a prey and a predator in both directions through contact or consumption.

This paper is organized in the following manner. Based on the available evidence demonstrating that resistant bacteria can spread between different populations, we establish differential equations model in Section 2. In Section 3, we derive the equilibrium points, and discuss the nonnegative points' existence conditions. In Section 4, we analyze the basic reproduction numbers. In this section, the threshold parameters are related to meaningful biological conditions. We then determine the stability conditions of the equilibrium points and prove them. In Section 5, we provide numerical results to discuss some interesting biological cases that our model is able to exhibit and the role of some key parameters in the system. We conclude by discussing the practical significance and application of the model.

\section{Model}

In this model, food animals consume antibiotic-containing feed over an extended period of time, and the bacteria evolve resistance to the antibiotics. This resistance can spread to humans via consumption or direct contact and then spread among the human population. Thus, both food animals and humans can be infected by the resistant bacteria. There are four populations in our model: $x_{1}(t)$ is the population size of the susceptible food animals at time $t . x_{2}(t)$ is the population size of the infected food animals at time $t . y_{1}(t)$ represents the amount of susceptible humans at time $t$, and $y_{2}(t)$ represents the amount of infected humans at time $t$.

To construct the model, we made the following assumptions.

(1) There is no recovery or immunity from resistant bacteria.

(2) The resistant bacteria can be transmitted vertically.

(3) $b$ is the growth rate of the food animals; $K$ is the carrying capacity of the food animals.

(4) $\beta_{1}\left(0<\beta_{1}<1\right)$ represents the conversion rate from susceptible food animals to infected animals. Since the quantities of the antibiotics added in feed are usually stationary, the conversion rate from susceptible food animals to infected animals is constant. The value of $\beta_{1}$ is only related to antibiotics in feed additives and is not related to $x_{2}$. To some extent, $\beta_{1}$ also represents the proportion of antibiotics in the 
feed. In this model, we do not consider the resistant bacteria's spread among food animals.

(5) To determine whether or not the food animals are infected is nearly impossible; however, resistance in the food animals does not affect the predation rate $p$. Humans reproduce at a certain rate after eating: $e p$ is the predator's reproduction rate, where $0<e<1$.

(6) $d$ is the death rate of infected animals; $d_{1}$ is the natural death rate of humans; $d_{2}$ is the human death rate due to infection by resistant bacteria, $d_{2}>d_{1}$.

(7) $\beta_{2}$ is the infection rate among humans and $\beta_{3}(0<$ $\left.\beta_{3}<1\right)$ is the infection rate from food animals to humans. Due to the cross-infection mechanism of resistant bacteria, the term $\beta_{3} x_{2} y_{1}$ characterizes the transmission dynamics $[63,64]$. The resistant bacteria's transmission in food animals is not considered.

Given the assumptions listed above, we obtain the following autonomous nonlinear differential equations:

$$
\begin{aligned}
& \dot{x}_{1}=b x_{1}\left(1-\frac{\left(x_{1}+x_{2}\right)}{K}\right)-b \beta_{1} x_{1}-p x_{1} y_{1}-p x_{1} y_{2}, \\
& \dot{x}_{2}=b \beta_{1} x_{1}-p x_{2} y_{1}-p x_{2} y_{2}-d x_{2}, \\
& \dot{y}_{1}=-d_{1} y_{1}-\beta_{2} y_{1} y_{2}+e p x_{1} y_{1}+e p x_{2} y_{1}-\beta_{3} x_{2} y_{1}, \\
& \dot{y}_{2}=-d_{2} y_{2}+\beta_{2} y_{1} y_{2}+e p x_{1} y_{2}+e p x_{2} y_{2}+\beta_{3} x_{2} y_{1} .
\end{aligned}
$$

To analyze the stability of the system, we first determine the equilibrium points.

\section{Equilibrium Points}

3.1. The Existence of Nonnegative Equilibrium Points. It is easy to obtain the four nonnegative equilibrium points of model (1). They are

$$
\begin{aligned}
& E_{0}(0,0,0,0), \\
& E_{1}\left(\frac{K d\left(1-\beta_{1}\right)}{\left(d+b \beta_{1}\right)}, \frac{K \beta_{1} b\left(1-\beta_{1}\right)}{\left(d+b \beta_{1}\right)}, 0,0\right), \\
& E_{2}\left(\tilde{x}_{1}, \tilde{x}_{2}, 0, \tilde{y}_{2}\right), \\
& E_{3}\left(x_{1}^{*}, x_{2}^{*}, y_{1}^{*}, y_{2}^{*}\right),
\end{aligned}
$$

where

$$
\begin{aligned}
& \tilde{x}_{1}=\frac{d_{2}\left(K e p d+K e p b-d_{2} b-K e p b \beta_{1}\right)}{\left(e p\left(K e p d+K e p b-d_{2} b\right)\right)}, \\
& \tilde{x}_{2}=\frac{K \beta_{1} d_{2} b}{\left(K e p d+K e p b-d_{2} b\right)}, \\
& \tilde{y}_{2}=\frac{b\left(K e p-K e p \beta_{1}-d_{2}\right)}{\left(e p^{2} K\right)}, \\
& x_{1}^{*}=\frac{X\left(K d+K b-b X-b \beta_{1} K\right)}{\left(b\left(X+K \beta_{1}\right)\right)}, \\
& x_{2}^{*}=X,
\end{aligned}
$$

$$
\begin{aligned}
& y_{1}^{*}= \frac{T}{\left(p \beta_{2} b\left(X+K \beta_{1}\right)\right)}, \\
& y_{2}^{*}=\left(-X d_{1} b-\beta_{3} X^{2} b-b d_{1} \beta_{1} K+K e p X d\right. \\
&\left.\quad-K \beta_{1} \beta_{3} X b+b K e p X\right) \\
& \cdot\left(\beta_{2} b\left(X+K \beta_{1}\right)\right)^{-1}, \\
& T=\beta_{2} b^{2} \beta_{1} K+p X d_{1} b+p X^{2} \beta_{3} b+p b \beta_{1} d_{1} K \\
&-e p^{2} X K d+p X K \beta_{1} \beta_{3} b-e p^{2} X K b \\
&- X d \beta_{2} b-X b^{2} \beta_{1} \beta_{2}-b^{2} \beta_{1}^{2} \beta_{2} K,
\end{aligned}
$$

and $X$ is the real positive root of the equation

$$
H_{1} x^{3}+H_{2} x^{2}+H_{3} x+H_{4}=0,
$$

where

$$
\begin{aligned}
H_{1}= & p \beta_{3} b\left(d_{1}-d_{2}\right), \\
H_{2}= & e p^{2} d_{2} b K+\beta_{2} e p d b K-e p^{2} d_{1} K d-\beta_{2} d d_{1} b \\
& +\beta_{2} e p d^{2} K-p d_{2} b d_{1}-d_{1} b^{2} \beta_{1} \beta_{2} \\
& +e p^{2} d_{2} K d-2 p d_{2} K b \beta_{1} \beta_{3}+2 p d_{1} K b \beta_{1} \beta_{3} \\
& -e p^{2} d_{1} b K+p d_{1}^{2} b+\beta_{2} b^{2} \beta_{1} e p K+\beta_{2} b \beta_{1} e p K d, \\
H_{3}= & -2 d_{1} b^{2} \beta_{1}^{2} \beta_{2} K+e p^{2} d_{2} K^{2} \beta_{1} b-e p^{2} d_{1} K^{2} \beta_{1} d \\
& -e p^{2} d_{1} K^{2} \beta_{1} b+e p^{2} d_{2} K^{2} \beta_{1} d-2 p d_{2} b \beta_{1} d_{1} K \\
& -\beta_{2} d d_{1} b \beta_{1} K+2 p d_{1}^{2} b \beta_{1} K+p d_{1} K^{2} \beta_{1}^{2} \beta_{3} b \\
& -\beta_{2} e p b^{2} K^{2} \beta_{1}-e p K^{2} d b \beta_{1} \beta_{2}+\beta_{2} b^{2} \beta_{1} K d_{1} \\
& +\beta_{2} \beta_{1}^{2} e p K^{2} b d-p d_{2} K^{2} b \beta_{1}^{2} \beta_{3}+\beta_{2} b^{2} \beta_{1}^{2} e p K^{2}, \\
H_{4}= & p d_{1}^{2} K^{2} \beta_{1}^{2} b+\beta_{2} b^{2} \beta_{1}^{2} K^{2} d_{1} \\
& -\beta_{2} b^{2} \beta_{1}^{3} K^{2} d_{1}-p d_{2} K^{2} \beta_{1}^{2} b d_{1} .
\end{aligned}
$$

In the next section, we will discuss the stability conditions of the different situations.

\section{Qualitative and Quantitative Analysis of the Model}

For each equilibrium point, we focus on whether the susceptible human can survive.

4.1. Qualitative Analysis of Model (1). First, we discuss the biological significance of the three threshold parameters that could be obtained from the stability analysis of the boundary equilibrium points. We will discuss the basic reproduction numbers in the following. 
We define

$$
\begin{aligned}
R_{0}^{1} & =\frac{e p K\left(1-\beta_{1}\right)\left(d+b \beta_{1}\right)}{\left(d_{1} d+b d_{1} \beta_{1}+b \beta_{1} K \beta_{3}-b K \beta_{3} \beta_{1}^{2}\right)}, \\
R_{0}^{2} & =\frac{e p K\left(1-\beta_{1}\right)}{d_{2}}, \\
R_{0}^{3} & =\frac{e p\left(\tilde{x}_{1}+\tilde{x}_{2}\right)}{\left(d_{1}+\beta_{2} \tilde{y}_{2}+\beta_{3} \tilde{x}_{2}\right)},
\end{aligned}
$$

where $\tilde{x}_{1}, \tilde{x}_{2}$, and $\tilde{y}_{2}$ are as defined in Section 3 .

Each of the threshold parameters has a clear and distinct biological meaning.

For $E_{1}$, the susceptible human growth rate is epK $\left(1-\beta_{1}\right)$ and the mortality rate is $\left(d_{1} d+b d_{1} \beta_{1}+b \beta_{1} K \beta_{3}-b K \beta_{3} \beta_{1}^{2}\right) /(d+$ $\left.b \beta_{1}\right)$, whereas the infected human growth rate is epK $\left(1-\beta_{1}\right)$ and the mortality rate is $d_{2}$.

$R_{0}^{1}<1$ implies that the susceptible predators will become extinct, while $R_{0}^{2}<1$ implies that the infected predators will become extinct. Hence, the combination of these two conditions results in $E_{1}$ being locally asymptotically stable.

For $E_{2}$, the susceptible human growth rate is $\operatorname{ep}\left(\tilde{x}_{1}+\tilde{x}_{2}\right)$ and the mortality rate is $\left(d_{1}+\beta_{2} \tilde{y}_{2}+\beta_{3} \tilde{x}_{2}\right)$. exist.

$R_{0}^{2}>1$ means that the infected predator population will

$R_{0}^{3}<1$ implies that the susceptible predator population will become extinct. Hence, the combination of these two conditions results in $E_{2}$ being locally asymptotically stable. Similarly, $R_{0}^{2}>1$ and $R_{0}^{3}>1$ are the locally asymptotically stable conditions for $E_{3}$.

These conclusions are summarized in Table 1.

4.2. Quantitative Analysis of Model (1). In order to determine the stability conditions of the equilibrium points, we calculated the Jacobi matrix $J=\left(J_{i j}\right)_{4 \times 4}$ for the equations, where

$$
\begin{aligned}
& J_{11}=b\left(1-\frac{\left(x_{1}+x_{2}\right)}{K}\right)-\frac{b x_{1}}{K}-b \beta_{1}-p y_{1}-p y_{2}, \\
& J_{12}=-\frac{b x_{1}}{K}, \quad J_{13}=-p x_{1}, \quad J_{14}=-p x_{1}, \\
& J_{21}=b \beta_{1}, \quad J_{22}=-p y_{1}-p y_{2}-d, \\
& J_{23}=-p x_{2}, \quad J_{24}=-p x_{2}, \\
& J_{31}=e p y_{1}, \quad J_{32}=e p y_{1}-\beta_{3} y_{1}, \\
& J_{33}=-d d_{1}-\beta_{2} y_{2}+e p x_{1}+e p x_{2}-\beta_{3} x_{2}, \\
& J_{34}=-\beta_{2} y_{1}, \quad J_{41}=e p y_{2}, \quad J_{42}=e p y_{2}+\beta_{3} y_{1}, \\
& J_{43}=\beta_{2} y_{2}+\beta_{3} x_{2}, \quad J_{44}=-d_{2}+\beta_{2} y_{1}+e p x_{1}+e p x_{2} .
\end{aligned}
$$

\subsection{Stability of the Boundary Equilibrium Point, $E_{0}$}

Theorem 1. The boundary equilibrium point $E_{0}(0,0,0,0)$ is always unstable.
TABLE 1: Asymptotic states for all equilibrium points.

\begin{tabular}{lcl}
\hline Equilibrium point & Feasible conditions & Dynamic behavior \\
\hline$E_{1}$ & $R_{0}^{1}<1 ; R_{0}^{2}<1$ & $\begin{array}{l}\text { Two types of } \\
\text { predator become } \\
\text { extinct }\end{array}$ \\
$E_{2}$ & $R_{0}^{2}>1 ; R_{0}^{3}<1$ & $\begin{array}{l}\text { Susceptible person } \\
\text { becomes extinct }\end{array}$ \\
$E_{3}$ & $R_{0}^{2}>1 ; R_{0}^{3}>1$ & All species coexist \\
\hline
\end{tabular}

Proof of Theorem 1. We obtain the Jacobi matrix for $E_{0}(0,0$, $0,0)$ as

$$
J_{0}=\left[\begin{array}{cccc}
b-b \beta_{1} & 0 & 0 & 0 \\
b \beta_{1} & 0 & 0 & 0 \\
0 & 0 & -d & 0 \\
0 & 0 & 0 & -d_{2}
\end{array}\right] .
$$

The eigenvalues of $J_{0}$ are

$$
\begin{array}{ll}
\lambda_{1}=0, & \lambda_{2}=b-b \beta_{1}, \\
\lambda_{3}=-d, & \lambda_{4}=-d_{2} .
\end{array}
$$

As a result of $\lambda_{2}=b-b \beta_{1}>0$, the boundary equilibrium point $E_{0}(0,0,0,0)$ is always unstable.

\subsection{Stability of the Boundary Equilibrium Point, $E_{1}$}

Theorem 2. If $R_{0}^{1}<1 ; R_{0}^{2}<1$, the boundary equilibrium point $E_{1}\left(K d\left(1-\beta_{1}\right) /\left(d+b \beta_{1}\right), K \beta_{1} b\left(1-\beta_{1}\right) /\left(d+b \beta_{1}\right), 0,0\right)$ is locally asymptotically stable.

The proof of Theorem 2 is provided in the Appendix A.

\subsection{Stability of the Boundary Equilibrium Point, $E_{2}$}

Theorem 3. If $R_{0}^{2}>1$ and $R_{0}^{3}<1$, the boundary equilibrium point $E_{2}\left(\tilde{x}_{1}, \tilde{x}_{2}, 0, \tilde{y}_{2}\right)$ is locally asymptotically stable, where $\tilde{x}_{1}$, $\tilde{x}_{2}$, and $\tilde{y}_{2}$ are as defined in Section 3.

The proof of Theorem 3 is provided in the Appendix B.

\subsection{Stability of the Interior Equilibrium Point, $E_{3}$}

Propose 1. If $R_{0}^{2}>1$ and $R_{0}^{3}>1$, the interior equilibrium point $E_{3}\left(x_{1}^{*}, x_{2}^{*}, y_{1}^{*}, y_{2}^{*}\right)$ is locally asymptotically stable.

We only numerically investigated the system's behavior around the interior feasible equilibrium point $E_{3}$ and provide the necessary numerical proof in the next section.

\subsection{Periodic Orbit of Model (1)}

Theorem 4. No periodic orbit of system (1) exists in

$$
\Omega=\left\{\left(x_{1}, x_{2}, y_{1}, y_{2}\right) \mid x_{1}>0, x_{2}>0, y_{1}>0, y_{2}>0\right\} \subset R^{4} \text {. }
$$

The proof of Theorem 4 is provided in the Appendix C. 
TABLE 2: Simulation results for fixed parameters: $b=0.8, K=15, p=0.45, d_{1}=0.02, \beta_{2}=0.005, e=0.05, d_{2}=0.07, d=0.01$, and $\beta_{3}=0.08$.

\begin{tabular}{lccc}
\hline Reproduction numbers' range & $\beta_{1}$ varied in a range & Stable equilibrium point & Dynamic behavior \\
\hline$R_{0}^{1}<1 ; R_{0}^{2}<1$ & $0.79<\beta_{1}<1$ & $E_{1}$ & Both predators become extinct \\
$R_{0}^{2}>1 ; R_{0}^{3}<1$ & $0.14<\beta_{1}<0.79$ & $E_{2}$ & Susceptible predators become extinct \\
$R_{0}^{2}>1 ; R_{0}^{3}>1$ & $0<\beta_{1}<0.14$ & $E_{3}$ & All the populations coexist \\
\hline
\end{tabular}

\section{Numerical Results and Parameter Analysis}

5.1. Numerical Results. Based on the analysis in the section above, conversion rate $\beta_{1}$ and predation rate $p$ that are the key parameters not only can affect all the basic reproduction numbers but also can be controlled by human. $\beta_{1}(0<$ $\left.\beta_{1}<1\right)$ represents the conversion rate from susceptible food animals to infected animals. In this paper, the conversion from susceptible food animals to infected animals is only due to the addition of antibiotics in the feed of food animals. In another word, even though without contacting, susceptible food animals can also convert to infected animals by eating feed with antibiotics. Therefore, the conversion rate $\beta_{1}$ depends on the amount of antibiotics used in feed. For the same feed, the amount of antibiotics added is larger, there will be more susceptible animals that convert to infected animals, and the conversion rate is bigger.

For further analysis of the steady-state of the equilibrium points and the parameter effects of $\beta_{1}$ and $p$, we illustrate some key numerical solutions.

When the parameter values are fixed at $b=0.8, K=15$, $p=0.45, d_{1}=0.02, \beta_{2}=0.005, e=0.05, d_{2}=0.07, d=0.01$, $\beta_{1}=0.1$, and $\beta_{3}=0.08$, we can calculate all of the equilibrium points as

$$
\begin{aligned}
& E_{1}(1.5,12,0,0), \\
& E_{2}(2.72,0.39,0,1.23), \\
& E_{3}(1.46,0.18,0.93,0.48) .
\end{aligned}
$$

The basic reproduction numbers are $R_{0}^{2}=4.34>1, R_{0}^{3}=$ $1.23>1$, and as seen in Propose 1 , the equilibrium point $E_{3}(1.46,0.18,0.93,0.48)$ is locally asymptotically stable.

The numerical proof of Propose 1 is as follows.

For the interior equilibrium point $E_{3}(1.46,0.18,0.93$, 0.48 ), the Jacobi matrix is

$$
J_{3}^{*}=\left[\begin{array}{cccc}
-0.08 & -0.08 & -0.66 & -0.66 \\
0.02 & -0.05 & 0 & -0.005 \\
0.01 & 0.09 & 0.02 & -0.03 \\
0.31 & 0.36 & 0.46 & -0.01
\end{array}\right] .
$$

The eigenvalues of $J_{3}^{*}$ are $\lambda_{1,2}=-0.05 \pm 0.15 i, \lambda_{3}=-0.63$, and $\lambda_{4}=-0.05$, and all the real parts of the eigenvalues are negative. Hence, the Routh-Hurwitz criteria are satisfied. Therefore, $E_{3}(1.46,0.18,0.93,0.48)$ is locally asymptotically stable.

5.2. Role of the Parameter $\beta_{1}$ in Model (1). $\beta_{1}\left(0<\beta_{1}<1\right)$ represents the conversion rate from susceptible food animals to infected animals and also represents the proportion of antibiotics in the feed. Therefore, as $\beta_{1}$ plays a major role in the outcome of the model, we will discuss its effect on the system. For convenience, our numerical results are summarized in Table 2 . The following initial values are used: $x_{1}(0)=0.5, x_{2}(0)=0.4$, and $y_{1}(0)=1, y_{2}(0)=2$.

In order to clearly show population dynamics for each case, three numerical results are given in the following for different values of $\beta_{1}$.

(a) $\beta_{1}=0.1$.

In this case, the basic reproduction number is $R_{0}^{2}=$ $4.34>1, R_{0}^{3}=1.23>1$ and so the interior equilibrium point $E_{3}(1.46,0.18,0.93,0.48)$ is locally asymptotically stable (the proof is provided above). The system tends toward the coexistence equilibrium (Figure 1). When increasing $\beta_{1}$ from zero to 0.14 , there is only a quantitative change, and the system's qualitative behavior remains the same. At this time, all the species coexist.

(b) $\beta_{1}=0.5$.

In this case, the basic reproduction number is $R_{0}^{2}=$ $2.41>1, R_{0}^{3}=0.40<1$ and so the equilibrium point $E_{2}(1.18,1.93,0,0.52)$ is locally asymptotically stable (Figure 2). The susceptible humans die out and the other three population types coexist in the system. This behavior remains for $0.14<\beta_{1}<0.79$.

(c) $\beta_{1}=0.96$.

In this case, we can calculate the basic reproduction number as $R_{0}^{1}=0.20<1, R_{0}^{2}=0.19<1$ and so the equilibrium $E_{1}(0.008,0.59,0,0)$ is locally asymptotically stable. Both of the human populations are extinct, and there are only food animals remaining in the system (Figure 3 ). In the wide range $0.79<\beta_{1}<$ 1 , the behavior of the system is qualitatively the same.

5.3. Role of the Parameter $p$ in Model (1). Besides the parameter $\beta_{1}$, we are also concerned about the role of the parameter $p$ in the system. $p$ is the predation rate in the model (1). As a result of the predator reproduction is dependent on its predation rate, so the change of predation rate affected both prey and predator. Owing to the predation rate can be controlled artificially, so predation rate is also a key parameter we care about. In this section, we will discuss the role of parameter $p$ in model (1). The conclusions are summarized in Table 3. The initial value are $x_{1}(0)=0.5, x_{2}(0)=0.4$, $y_{1}(0)=1$, and $y_{2}(0)=2$, and the parameter values except 

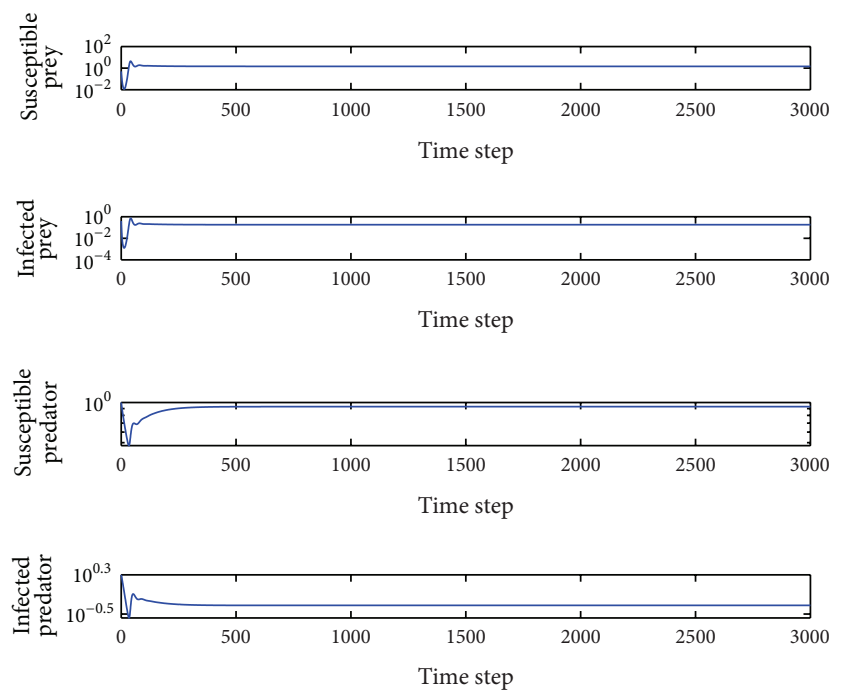

Figure 1: Evolution of the system populations for $\beta_{1}=0.1$; other parameters are as defined in Table 2 .
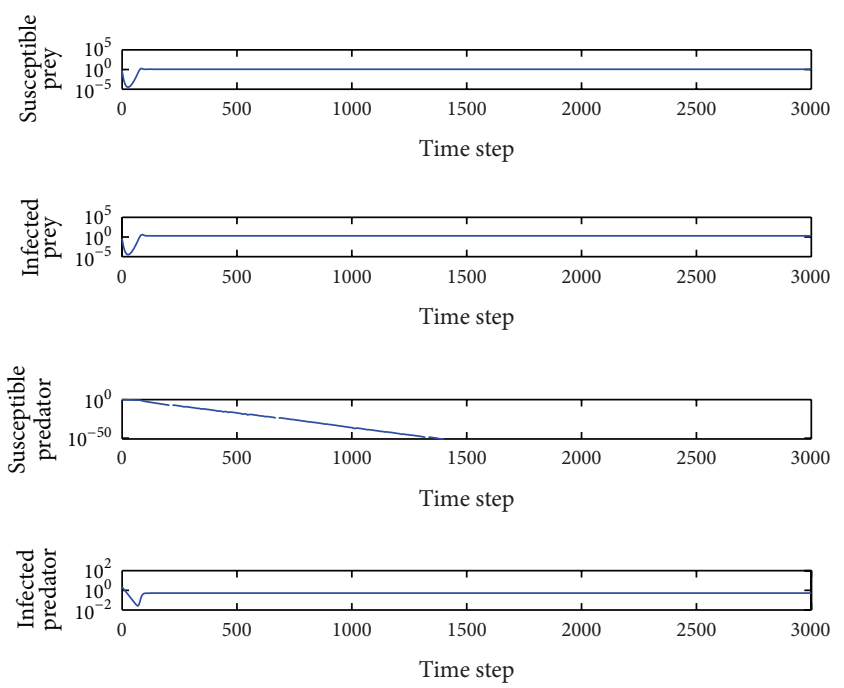

FIGURE 2: Evolution of the system populations for $\beta_{1}=0.5$; other parameters are as defined in Table 2 .

$p$ are $b=0.8, K=15, \beta_{1}=0.1, d_{1}=0.02, \beta_{2}=0.005$, $e=0.05, d_{2}=0.07, d=0.01$, and $\beta_{3}=0.08$.

We describe the influence of predation rate on the system under three different situations.

(a) $p=0.05$.

In this case, the basic reproduction number are $R_{0}^{1}=$ $0.03<1, R_{0}^{2}=0.48<1$, and the equilibrium $E_{1}(1.5,12,0,0)$ is locally asymptotically stable. Both of the human populations are extinct, and there are only food animals remaining in the system (Figure 4). It is similar to the situation in Figure 3. In the wide range $0<p<0.104$, the behavior of the system is qualitatively the same.
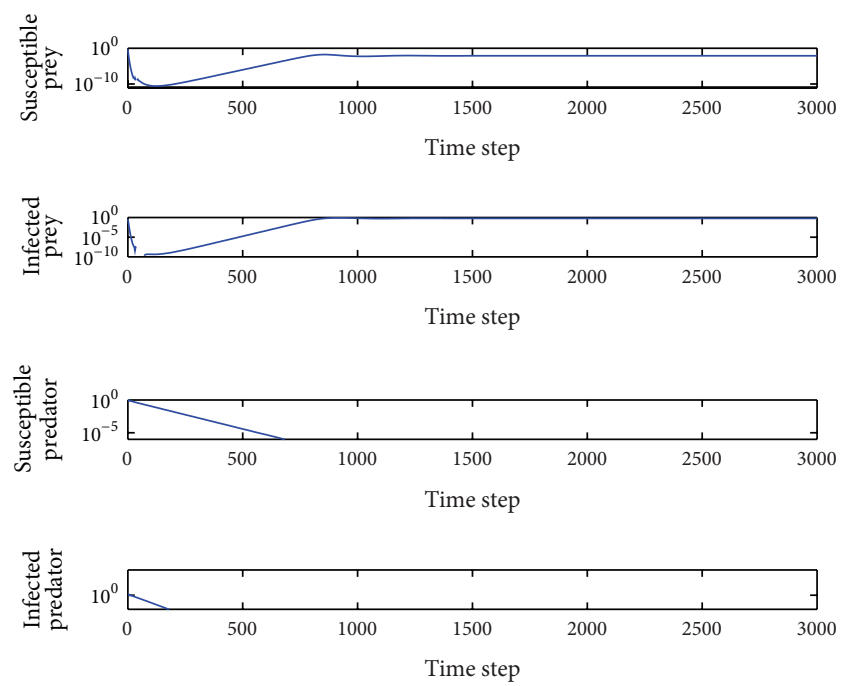

FIGURE 3: Evolution of the system populations for $\beta_{1}=0.96$; other parameters are as defined in Table 2.

(b) $p=0.25$.

In this case, the basic reproduction numbers are $R_{0}^{2}=$ $2.41>1, R_{0}^{3}=0.71<1$ and so the equilibrium point $E_{2}(1.18,1.93,0,0.52)$ is locally asymptotically stable (Figure 5). It is similar to the situation in Figure 2. The susceptible humans die out and the other three population types coexist in the system. This behavior remains for $0.104<p<0.351$.

(c) $p=0.55$.

In this case, the basic reproduction numbers are $R_{0}^{2}=$ $5.30>1, R_{0}^{3}=1.41>1$ and so the interior equilibrium point $E_{3}(1.00,0.12,0.94,0.26)$ is locally asymptotically stable. The system tends toward the coexistence equilibrium (Figure 6). It is similar to the situation in Figure 1. When increasing $p$ from 0.351 to 1 , there is only a quantitative change, and the system's qualitative behavior remains the same. At this time, all the species coexist.

5.4. Role of the Parameters $\beta_{1}$ and $p$ in the System. In the following section, we discuss the effect of $\beta_{1}$ and $p$ together, while maintaining the other parameters fixed as defined in Table 3 . We observed the dynamics when changing the parameters $\beta_{1}$ and $p$ between 0 and 1 . The resulting image is shown in Figure 7, in which three different regions were obtained after 3000 time steps, which are indicated by different colors.

In region $\mathrm{A}$, four populations can coexist. In region $\mathrm{B}$, the susceptible humans die out, and the other populations coexist. In region $\mathrm{C}$, there are only food animals in the system.

Based on the conclusion shown in Table 1, the three regions need to meet the following conditions:

region $\mathrm{A}$ :

$$
R_{0}^{2}>1, \quad R_{0}^{3}>1 \Longleftrightarrow p<\frac{1}{\left(10.7\left(1-\beta_{1}\right)\right)},
$$


TABLE 3: Simulation results for fixed parameters: $b=0.8, K=15, \beta_{1}=0.1, d_{1}=0.02, \beta_{2}=0.005, e=0.05, d_{2}=0.07, d=0.01$, and $\beta_{3}=0.08$.

\begin{tabular}{lccc}
\hline Reproduction numbers' range & $p$ varied in a range & Stable equilibrium point & Dynamic behavior \\
\hline$R_{0}^{1}<1 ; R_{0}^{2}<1$ & $0<p<0.104$ & $E_{1}$ & Both predators become extinct \\
$R_{0}^{2}>1 ; R_{0}^{3}<1$ & $0.104<p<0.351$ & $E_{2}$ & Susceptible predators become extinct \\
$R_{0}^{2}>1 ; R_{0}^{3}>1$ & $0.351<p<1$ & $E_{3}$ & All the populations coexist \\
\hline
\end{tabular}
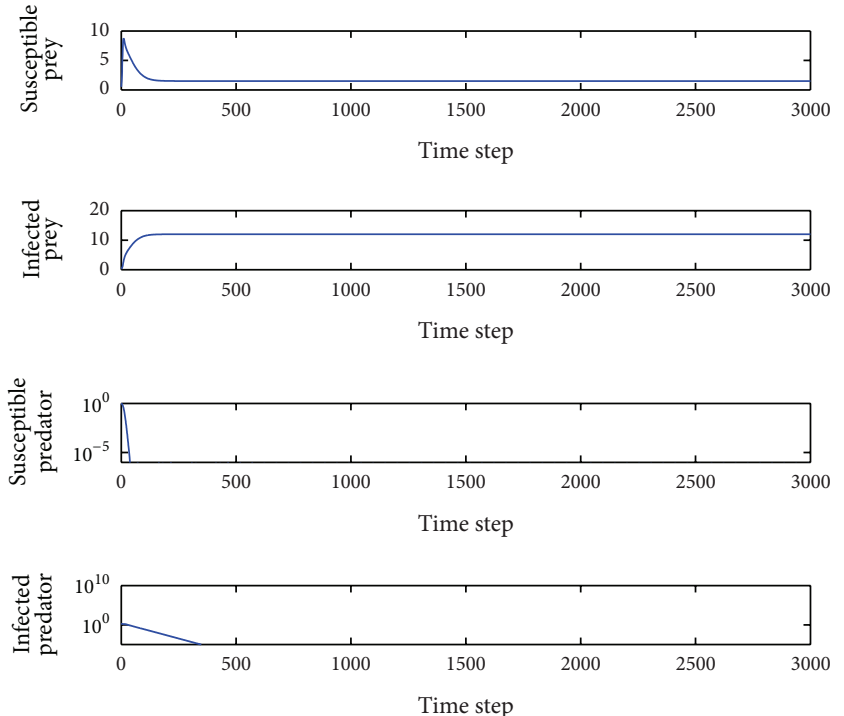

FIGURE 4: Evolution of the system populations for $p=0.05$; other parameters are as defined in Table 3.
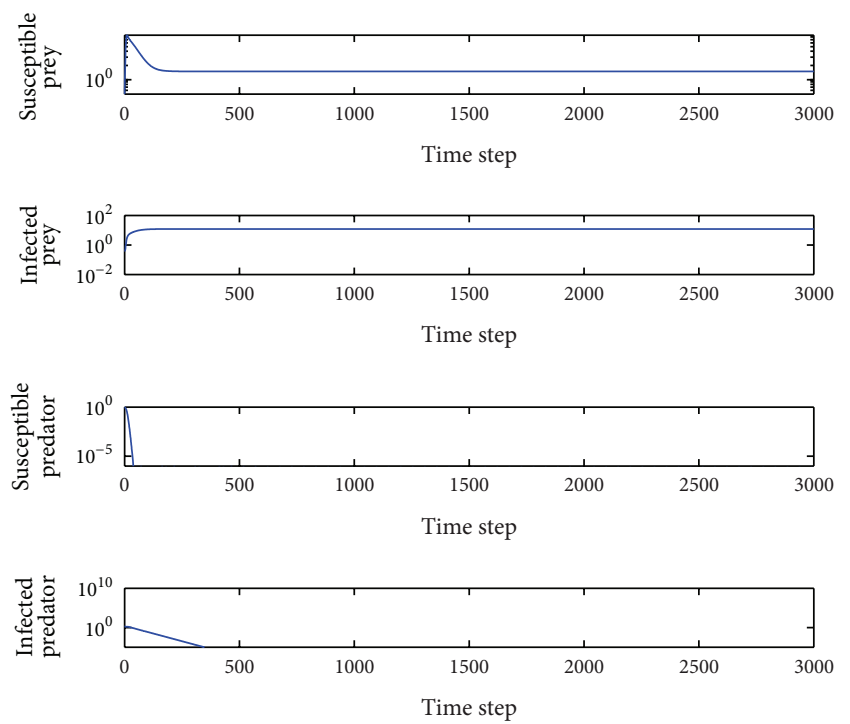

Figure 5: Evolution of the system populations for $p=0.25$; other parameters are as defined in Table 3.

$$
\begin{gathered}
\text { region B: } \\
R_{0}^{2}>1, \quad R_{0}^{3}<1 \Longleftrightarrow p>\frac{1}{\left(10.7\left(1-\beta_{1}\right)\right)}, \\
\beta_{1}>\frac{\left(0.469\left(p^{2}-0.081 p+0.008\right)(p-0.091)\right)}{((p+0.0035) p)},
\end{gathered}
$$
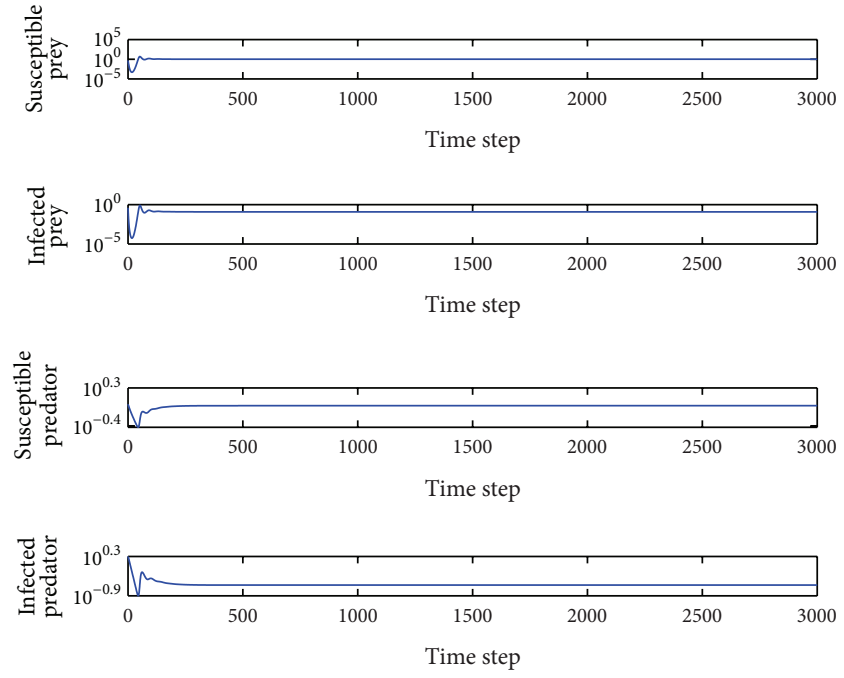

FIGURE 6: Evolution of the system populations for $p=0.55$; other parameters are as defined in Table 3.

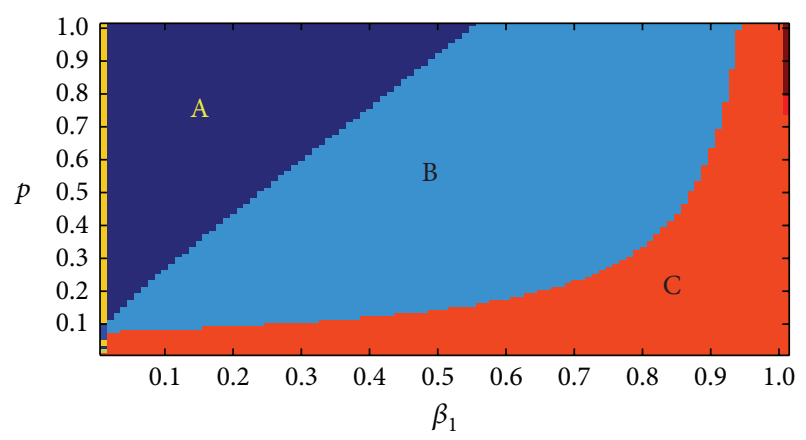

FIgURE 7: Changing $\beta_{1}$ and $p$ while maintaining the other parameters and the initial values fixed as defined in Table 3 , three regions were obtained after 3000 time steps.

region $\mathrm{C}$ :

$$
\begin{aligned}
R_{0}^{1}<1, \quad R_{0}^{2}<1 \\
\\
\Longleftrightarrow \beta_{1}<\frac{\left(0.469\left(p^{2}-0.081 p+0.008\right)(p-0.091)\right)}{((p+0.0035) p)} .
\end{aligned}
$$


At the same time, the divide lines of the three regions can be calculated. Divide line of region A and region B:

$$
\begin{aligned}
R_{0}^{3} & =1 \\
& \Longleftrightarrow \beta_{1}=\frac{\left(0.469\left(p^{2}-0.081 p+0.008\right)(p-0.091)\right)}{((p+0.0035) p)} .
\end{aligned}
$$

Divide line of region $\mathrm{B}$ and region $\mathrm{C}$ :

$$
R_{0}^{2}=1 \Longleftrightarrow p=\frac{1}{\left(10.7\left(1-\beta_{1}\right)\right)} .
$$

\section{Discussion}

Three steady states were obtained from the model described in this paper: in the first state, all populations coexist in the system; in the second state, there are no susceptible humans in the system, but the others coexist; and in the third state, only food animals remain in the system. Among these cases, only the first is a favorable outcome for humans. As shown in Figure 4, for a fixed $p$, as the value of $\beta_{1}$ gets bigger, the possibility of coexistence will be smaller, even becoming impossible. Because the predation rate $p$ cannot be easy controlled, in order to achieve the first state, we clearly need to reduce the value of $\beta_{1}$.

On the other hand, the recent increase in society's attention to food safety suggests that food animals that consume antibiotic-containing feed might be consumed less by humans, which would result in a decrease in the predation rate $p$. At this point, to achieve coexistence, a smaller value of $\beta_{1}$ is needed. Therefore, proper control of the amount of feed antibiotics used is necessary.
How can the value of $\beta_{1}$ be reasonably controlled? When $R_{0}^{2}>1$ and $R_{0}^{3}>1$, the four species can coexist, and we obtain the relationship between $\beta_{1}$ and $p$ shown in Section 5.2. Therefore, we can adjust the value of $\beta_{1}$ to satisfy the condition of coexistence.

As the value of $p$ is not easy to measure and control, we instead need to focus on controlling the amount of antibiotics in the feed. As the value of $p$ is expected to decrease with increased public awareness of food safety, controlling the value of $\beta_{1}$ will become increasingly important. If we ignore the amount of antibiotics used in feed, and the predation rate continues to decrease, the antibiotic use rate could potentially surpass our "safety margin" without notice. In such a scenario, the human population will be predominantly susceptible and could become extinct in the most extreme case. In order to avoid this situation, proper control of the amount of antibiotics used in feed for food animals is extremely necessary.

\section{Appendices}

\section{A. Proof of Theorem 2}

Proof. Let

$$
\begin{aligned}
R_{0}^{1} & =\frac{e p K\left(1-\beta_{1}\right)\left(d+b \beta_{1}\right)}{\left(d d_{1}+d_{1} b \beta_{1}+\beta_{3} b \beta_{1} K-\beta_{3} b \beta_{1}^{2} K\right)}, \\
R_{0}^{2} & =\frac{e p K\left(1-\beta_{1}\right)}{d_{2}} .
\end{aligned}
$$

For the equilibrium point $E_{1}\left(K d\left(1-\beta_{1}\right) /\left(d+b \beta_{1}\right), K \beta_{1} b(1-\right.$ $\left.\left.\beta_{1}\right) /\left(d+b \beta_{1}\right), 0,0\right)$, the Jacobi matrix is

$$
J_{1}=\left[\begin{array}{cccc}
\frac{b d\left(\beta_{1}-1\right)}{\left(d+b \beta_{1}\right)} & \frac{b d\left(\beta_{1}-1\right)}{\left(d+b \beta_{1}\right)} & \frac{p K d\left(\beta_{1}-1\right)}{\left(d+b \beta_{1}\right)} & \frac{p K d\left(\beta_{1}-1\right)}{\left(d+b \beta_{1}\right)} \\
b \beta_{1} & -d & \frac{p b \beta_{1} K\left(\beta_{1}-1\right)}{\left(d+b \beta_{1}\right)} & \frac{p b \beta_{1} K\left(\beta_{1}-1\right)}{\left(d+b \beta_{1}\right)} \\
0 & 0 & J_{33} & 0 \\
0 & 0 & \frac{\beta_{1} b \beta_{3} K\left(1-\beta_{1}\right)}{\left(d+b \beta_{1}\right)} & -d_{2}+K e p-e p \beta_{1} K
\end{array}\right],
$$

where $J_{33}=-\left(d d_{1}+d_{1} b \beta_{1}-e p K d+e p K d \beta_{1}-e p b \beta_{1} K+\right.$ $\left.e p b \beta_{1}^{2} K+\beta_{3} b \beta_{1} K-\beta_{3} b \beta_{1}^{2} K\right) /\left(d+b \beta_{1}\right)$.

The eigenvalues of $J_{1}$ are

$$
\begin{aligned}
\lambda_{1}=- & \left(d d_{1}+d_{1} b \beta_{1}-e p K d+e p K d \beta_{1}-e p b \beta_{1} K\right. \\
& \left.+e p b \beta_{1}^{2} K+\beta_{3} b \beta_{1} K-\beta_{3} b \beta_{1}^{2} K\right) \\
& \cdot\left(d+b \beta_{1}\right)^{-1}
\end{aligned}
$$

$$
\lambda_{2}=-d_{2}+K e p-e p \beta_{1} K
$$$$
\lambda_{3}=\left(-b d-d^{2}+\left(b^{2} d^{2}-2 b d^{3}+d^{4}\right.\right.
$$$$
+4 b d^{3} \beta_{1}+8 b^{2} \beta_{1}^{2} d^{2}
$$$$
\left.\left.-8 b^{2} \beta_{1} d^{2}+4 b^{3} \beta_{1}^{3} d-4 b^{3} \beta_{1}^{2} d\right)^{1 / 2}\right),
$$

$$
\cdot\left(2\left(d+b \beta_{1}\right)\right)^{-1}
$$




$$
\begin{aligned}
\lambda_{4}=\left(-b d-d^{2}-\left(b^{2} d^{2}-2 b d^{3}+d^{4}+4 b d^{3} \beta_{1}\right.\right. \\
+8 b^{2} \beta_{1}^{2} d^{2}-8 b^{2} \beta_{1} d^{2} \\
\left.\left.+4 b^{3} \beta_{1}^{3} d-4 b^{3} \beta_{1}^{2} d\right)^{1 / 2}\right) \\
\cdot\left(2\left(d+b \beta_{1}\right)\right)^{-1} .
\end{aligned}
$$

According to $R_{0}^{1}<1, R_{0}^{2}<1$, we get $\lambda_{1}<0, \lambda_{2}<0$, and

$$
\begin{gathered}
\left(b d+d^{2}\right)^{2}-\left(b^{2} d^{2}-2 b d^{3}+d^{4}+4 b d^{3} \beta_{1}+8 b^{2} \beta_{1}^{2} d^{2}\right. \\
\left.-8 b^{2} \beta_{1} d^{2}+4 b^{3} \beta_{1}^{3} d-4 b^{3} \beta_{1}^{2} d\right) \\
=4 b d\left(1-\beta_{1}\right)\left(d+b \beta_{1}\right)^{2}>0 .
\end{gathered}
$$

The real parts of $\lambda_{3}, \lambda_{4}$ are both negative. Using the RouthHurwitz criteria, the equilibrium point $E_{1}\left(K d\left(1-\beta_{1}\right) /(d+\right.$ $\left.\left.b \beta_{1}\right), K b \beta_{1}\left(1-\beta_{1}\right) /\left(d+b \beta_{1}\right), 0,0\right)$ is locally asymptotically stable.

\section{B. Proof of Theorem 3}

Proof. For the equilibrium point $E_{2}\left(\widetilde{x}_{1}, \widetilde{x}_{2}, 0, \widetilde{y}_{2}\right)$, where

$$
\tilde{x}_{1}=\frac{d_{2}\left(K e p d+K e p b-d_{2} b-K e p b \beta_{1}\right)}{\left(e p\left(K e p d+K e p b-d_{2} b\right)\right)},
$$

$$
J_{2}=\left[\begin{array}{cc}
-\frac{b d_{2} B}{A e p K} & -\frac{b d_{2} B}{A e p K} \\
b \beta_{1} & -\frac{(C+K e p d)}{K e p} \\
0 & 0 \\
\frac{C}{p K} & \frac{C}{p K}
\end{array}\right.
$$

$$
\left.\begin{array}{cc}
-\frac{d_{2} B}{A e} & -\frac{d_{2} B}{A e} \\
-\frac{p K \beta_{1} d_{2} b}{A} & -\frac{p K \beta_{1} d_{2} b}{A} \\
-\frac{\left(A C \beta_{2}+\beta_{3} K^{2} \beta_{1} d_{2} b e p^{2}\right)}{\left(e p^{2} K A\right)}+d_{2}-d_{1} & 0 \\
\frac{\left(A C \beta_{2}+\beta_{3} K^{2} \beta_{1} d_{2} b e p^{2}\right)}{\left(e p^{2} K A\right)} & 0
\end{array}\right] .
$$

For the convenient expression, let expressed by

$$
\begin{gathered}
\tilde{x}_{1}=\frac{d_{2} B}{(A e p)}, \quad \tilde{x}_{2}=\frac{K \beta_{1} d_{2} b}{A}, \\
\tilde{y}_{2}=\frac{C}{\left(e p^{2} K\right)}, \\
R_{0}^{3}=\frac{d_{2} A e p^{2} K}{\left(A C \beta_{2}+\beta_{3} K^{2} \beta_{1} d_{2} b e p^{2}+d_{1} A e p^{2} K\right)} .
\end{gathered}
$$

Because of $R_{0}^{3}<1$, it is easy to get $M_{1}>0$.

The Jacobi matrix is

$$
\begin{aligned}
& A=K e p d+K e p b-d_{2} b, \\
& B=K e p d+K e p b-d_{2} b-K e p b \beta_{1}, \\
& C=b\left(K e p-K e p \beta_{1}-d_{2}\right), \\
& M=A(K e p C+B b)>0, \\
& M_{1}=A C \beta_{2}+\beta_{3} K^{2} \beta_{1} d_{2} b e p^{2}-\left(d_{2}-d_{1}\right) A e p^{2} K .
\end{aligned}
$$

So the equilibrium point and reproduction number can be

The characteristic equation is

$$
\begin{aligned}
& A_{3}=\frac{d_{2}\left(M_{1} M+A^{2} B C e p^{2} K\right)}{\left(K^{3} e^{3} p^{4} A^{2}\right)}>0, \\
& A_{4}=\frac{M_{1} A B C d_{2}}{\left(K^{3} e^{3} p^{4} A^{2}\right)}>0,
\end{aligned}
$$$$
\lambda^{4}+A_{1} \lambda^{3}+A_{2} \lambda^{2}+A_{3} \lambda+A_{4}=0,
$$

where

$$
\begin{aligned}
& A_{1} A_{2}-A_{3} \\
& \quad=((b+d)
\end{aligned}
$$$$
A_{1}=\frac{\left(M_{1}+\operatorname{Kep}^{2} B(b+d)\right)}{\left(A e p^{2} K\right)}>0,
$$$$
A_{2}=\frac{\left(M_{1} B K e p(b+d)+p d_{2} M A\right)}{\left(K^{2} e^{2} p^{3} A^{2}\right)}>0 \text {, }
$$ 


$$
\begin{aligned}
& \cdot\left(K^{2} e^{2} p^{4} A^{3}\right)^{-1}>0, \\
& A_{1} A_{2} A_{3}-A_{3}^{2}-A_{1}^{2} A_{4} \\
&=d_{2} B A\left(b d_{2} C+(b+d) B b\right) \\
& \cdot\left(d_{2} B e p^{4} K C A^{3}+M_{1} d_{2} M p^{2} A+M_{1}^{3}\right. \\
&\left.\quad+M_{1}^{2} B K e p^{2} d+M_{1}^{2} B K e b p^{2}\right) \\
& \cdot\left(K^{5} e^{5} p^{8} A^{5}\right)^{-1}>0 .
\end{aligned}
$$

Using the Routh-Hurwitz criteria, the equilibrium point $E_{2}\left(\widetilde{x}_{1}, \widetilde{x}_{2}, 0, \widetilde{y}_{2}\right)$ is locally asymptotically stable.

\section{Proof of Theorem 4}

Proof. If a periodic orbit of model (1) in $\Omega$ exists, its projection onto some two-dimensional subspace of $R^{4}$ should also be periodic. Therefore, we have to investigate if any periodic solution exists or not an all two-dimensional subspace. There are six different two-dimensional subsystems of (1).

For the subsystem,

$$
\begin{aligned}
& \dot{x}_{1}=b x_{1}\left(1-\frac{x_{1}+x_{2}}{K}\right)-b \beta_{1} x_{1}-p x_{1} y_{1}-p x_{1} y_{2}, \\
& \dot{x}_{2}=b \beta_{1} x_{1}-p x_{2} y_{1}-p x_{2} y_{2}-d x_{2} .
\end{aligned}
$$

We choose the Dulac function $B_{1}=1 / x_{1} x_{2}$, to evaluate the following expression:

$$
\frac{\partial\left(B_{1} \dot{x}_{1}\right)}{\partial x_{1}}+\frac{\partial\left(B_{1} \dot{x}_{2}\right)}{\partial x_{2}}=-\frac{b}{K x_{2}}-\frac{b \beta_{1}}{x_{2}^{2}}<0 .
$$

For the other five subsystems, we choose the Dulac functions

$$
\begin{array}{ll}
B_{2}=\frac{1}{x_{2} y_{1}}, & B_{3}=\frac{1}{y_{1} y_{2}}, \quad B_{4}=\frac{1}{x_{1} y_{1}}, \\
B_{5}=\frac{1}{x_{1} y_{2}}, & B_{6}=\frac{1}{x_{2} y_{2}} ;
\end{array}
$$

similarly

$$
\begin{gathered}
\frac{\partial\left(B_{2} \dot{x}_{2}\right)}{\partial x_{2}}+\frac{\partial\left(B_{2} \dot{y}_{1}\right)}{\partial y_{1}}=-\frac{b \beta_{1} x_{1}}{x_{2}^{2} y_{1}}<0, \\
\frac{\partial\left(B_{3} \dot{y}_{1}\right)}{\partial y_{1}}+\frac{\partial\left(B_{3} \dot{y}_{2}\right)}{\partial y_{2}}=-\frac{\beta_{3} x_{2}}{y_{2}^{2}}<0, \\
\frac{\partial\left(B_{4} \dot{x}_{1}\right)}{\partial x_{1}}+\frac{\partial\left(B_{4} \dot{y}_{1}\right)}{\partial y_{1}}=-\frac{b}{K y_{1}}<0, \\
\frac{\partial\left(B_{5} \dot{x}_{1}\right)}{\partial x_{1}}+\frac{\partial\left(B_{5} \dot{y}_{2}\right)}{\partial y_{2}}=-\frac{b}{K y_{2}}-\frac{\beta_{3} x_{2} y_{1}}{x_{1} y_{2}^{2}}<0, \\
\frac{\partial\left(B_{6} \dot{x}_{2}\right)}{\partial x_{2}}+\frac{\partial\left(B_{6} \dot{y}_{2}\right)}{\partial y_{2}}=-\frac{b \beta_{1} x_{1}}{x_{2}^{2} y_{2}}-\frac{\beta_{3} y_{1}}{x_{2}^{2}}<0 .
\end{gathered}
$$

Now, using the Bendixson-Dulac negative criterion, no periodic solution in these two dimensions can exist. Therefore, the solution of (1) in $R^{4}$ also cannot oscillate persistently.

\section{Conflict of Interests}

The authors declare that there is no conflict of interests regarding the publication of this paper.

\section{References}

[1] E. L. Stokstad and T. H. Jukes, "The multiple nature of the animal protein factor," The Journal of Biological Chemistry, vol. 180, no. 2, pp. 647-654, 1949.

[2] R. L. Preston, "The role of animal drugs in food animal production," 1988.

[3] T. G. Nagaraja and M. M. Chengappa, "Liver abscesses in feedlot cattle: a review," Journal of Animal Science, vol. 76, no. 1, pp. 287298, 1998.

[4] H. R. Gaskins, C. T. Collier, and D. B. Anderson, "Antibiotics as growth promotants: mode of action," Animal Biotechnology, vol. 13, no. 1, pp. 29-42, 2002.

[5] D. McClary and G. Vogel, "Effect of timing of tilmicosin metaphylaxis on control of bovine respiratory disease and performance in feeder cattle," The Bovine Practitioner, no. 33, pp. 155-161, 1999.

[6] B. Flemming, E. Hanne-Dorthe, L. Monnet Dominique et al., DANMAP 2000: Consumption of Antimicrobial Agents and Occurence of Antimicrobial Resistance in Bacteria from Food Animals, Foods and Humans in Denmark, Danish Veterinary Institute, 2001.

[7] B. Flemming, H.-D. Emborg, A. Sigrid et al., "DANMAP 97: consumption of antimicrobial agents and occurrence of antimicrobial resistance in bacteria from food animals, food and humans in Danmark," 1998.

[8] F. Bager, DANMAP 98-Consumption of antimicrobial agents and occurrence of antimicrobial in bacteria from food animals, food and humans in Denmark, 1999.

[9] F. Bager, DANMAP 99-Consumption of Antimicrobial Agents and Occurrence of Antimicrobial Resistance in Bacteria from Food Animals, Food and Humans in Denmark, Statens Serum Institut, Danish Veterinary and Food Administration, Danish Medicines Agency, Danish Veterinary Laboratory, Copenhagen, Denmark, 2000.

[10] F. Bager, H. D. Emborg, F. M. Aarestrup et al., "The Danish experience following the ban of antimicrobial growth promoters: trends in microbial resistance and antimicrobial use," in Proceedings of Alltech's 18th Annual Symposium: From Niche Markets to Mainstream, Lexington, Ky, USA, 2002.

[11] V. Perreten, F. Schwarz, L. Cresta, M. Boeglin, G. Dasen, and M. Teuber, "Antibiotic resistance spread in food," Nature, vol. 389, no. 6653, pp. 801-802, 1997.

[12] M. Teuber, "Spread of antibiotic resistance with food-borne pathogens," Cellular and Molecular Life Sciences, vol. 56, no. 910, pp. 755-763, 1999.

[13] V. J. Harwood, M. Brownell, W. Perusek, and J. E. Whitlock, "Vancomycin-resistant Enterococcus spp. Isolated from Wastewater and chicken feces in the United States," Applied and Environmental Microbiology, vol. 67, no. 10, pp. 4930-4933, 2001.

[14] L. A. Devriese, M. Ieven, H. Goossens et al., "Presence of vancomycin-resistant enterococci in farm and pet animals," 
Antimicrobial Agents and Chemotherapy, vol. 40, no. 10, pp. 2285-2287, 1996.

[15] D. J. P. Mallon, J. E. Corkill, S. M. Hazel et al., "Excretion of vancomycin-resistant enterococci by wild mammals," Emerging Infectious Diseases, vol. 8, no. 6, pp. 636-638, 2002.

[16] A. E. van den Bogaard, N. London, C. Driessen, and E. E. Stobberingh, "Antibiotic resistance of faecal Escherichia coli in poultry, poultry farmers and poultry slaughterers," Journal of Antimicrobial Chemotherapy, vol. 47, no. 6, pp. 763-771, 2001.

[17] S. M. Donabedian, L. A. Thal, E. Hershberger et al., "Molecular characterization of gentamicin-resistant Enterococci in the United States: Evidence of spread from animals to humans through food," Journal of Clinical Microbiology, vol. 41, no. 3, pp. 1109-1113, 2003.

[18] A. D. Anderson, J. M. Nelson, S. Rossiter, and F. J. Angulo, “ Public health consequences of use of antimicrobial agents in food animals in the United States," Microbial Drug Resistance, vol. 9, no. 4, pp. 373-379, 2003.

[19] I. Phillips, M. Casewell, T. Cox et al., "Does the use of antibiotics in food animals pose a risk to human health? A critical review of published data," Journal of Antimicrobial Chemotherapy, vol. 53, no. 1, pp. 28-52, 2004.

[20] L. Tollefson and M. A. Miller, "Antibiotic use in food animals: controlling the human health impact," Journal of AOAC International, vol. 83, no. 2, pp. 245-254, 2000.

[21] C. T. Bergstrom, M. Lo, and M. Lipsitch, "Ecological theory suggests that antimicrobial cycling will not reduce antimicrobial resistance in hospitals," Proceedings of the National Academy of Sciences of the United States of America, vol. 101, no. 36, pp. 13285-13290, 2004.

[22] S. Bonhoeffer, M. Lipsitch, and B. R. Levin, "Evaluating treatment protocols to prevent antibiotic resistance," Proceedings of the National Academy of Sciences of the United States of America, vol. 94, no. 22, pp. 12106-12111, 1997.

[23] R. D. Kouyos, P. A. zur Wiesch, and S. Bonhoeffer, "On being the right size: the impact of population size and stochastic effects on the evolution of drug resistance in hospitals and the community," PLoS Pathogens, vol. 7, no. 4, Article ID e1001334, 2011.

[24] G. F. Webb, E. M. C. D’Agata, P. Magal, and S. Ruan, "A model of antibiotic-resistant bacterial epidemics in hospitals," Proceedings of the National Academy of Sciences of the United States of America, vol. 102, no. 37, pp. 13343-13348, 2005.

[25] H. R. Sun, X. Lu, and S. Ruan, "Qualitative analysis of models with different treatment protocols to prevent antibiotic resistance," Mathematical Biosciences, vol. 227, no. 1, pp. 56-67, 2010.

[26] D. J. Austin and R. M. Anderson, "Studies of antibiotic resistance within the patient, hospitals and the community using simple mathematical models," Philosophical Transactions of the Royal Society B: Biological Sciences, vol. 354, no. 1384, pp. 721738, 1999.

[27] G. L. French, "The continuing crisis in antibiotic resistance," International Journal of Antimicrobial Agents, vol. 36, no. 3, pp. S3-S7, 2010.

[28] R. A. Weinstein, M. J. M. Bonten, D. J. Austin, and M. Lipsitch, "Understanding the spread of antibiotic resistant pathogens in hospitals: mathematical models as tools for control," Clinical Infectious Diseases, vol. 33, no. 10, pp. 1739-1746, 2001.

[29] D. J. Austin, M. J. M. Bonten, R. A. Weinstein, S. Slaughter, and R. M. Anderson, "Vancomycin-resistant enterococci in intensive-care hospital settings: transmission dynamics, persistence, and the impact of infection control programs," Proceedings of the National Academy of Sciences of the United States of America, vol. 96, no. 12, pp. 6908-6913, 1999.

[30] A. J. Lotka, "Analytical note on certain rhythmic relations in organic systems," Proceedings of the National Academy of Sciences of the United States of America, vol. 6, no. 7, pp. 410415,1920

[31] K. P. Hadeler and H. I. Freedman, "Predator-prey populations with parasitic infection," Journal of Mathematical Biology, vol. 27, no. 6, pp. 609-631, 1989.

[32] E. Venturino, "The influence of diseases on Lotka-Volterra systems," The Rocky Mountain Journal of Mathematics, vol. 24, no. 1, pp. 381-402, 1994.

[33] B. Edward and T. O. Carroll, "Modeling the role of viral disease in recurrent phytoplankton blooms," Journal of Mathematical Biology, vol. 32, no. 8, pp. 857-863, 1994.

[34] E. Venturino, "The effects of diseases on competing species," Mathematical Biosciences, vol. 174, no. 2, pp. 111-131, 2001.

[35] J. Chattopadhyay, S. Pal, and A. El Abdllaoui, "Classical predator-prey system with infection of prey population? A mathematical model," Mathematical Methods in the Applied Sciences, vol. 26, no. 14, pp. 1211-1222, 2003.

[36] H. W. Hethcote, W. Wang, L. Han, and Z. Ma, "A predatorprey model with infected prey," Theoretical Population Biology, vol. 66, no. 3, pp. 259-268, 2004.

[37] L. Han, Z. Ma, and H. W. Hethcote, "Four predator prey models with infectious diseases," Mathematical and Computer Modelling, vol. 34, no. 7-8, pp. 849-858, 2001.

[38] J. Chattopadhyay, R. R. Sarkar, and G. Ghosal, "Removal of infected prey prevent limit cycle oscillations in an infected preypredator system - a mathematical study," Ecological Modelling, vol. 156, no. 2-3, pp. 113-121, 2002.

[39] K. P. Das, S. Chatterjee, and J. Chattopadhyay, "Disease in prey population and body size of intermediate predator reduce the prevalence of chaos-conclusion drawn from Hastings-Powell model," Ecological Complexity, vol. 6, no. 3, pp. 363-374, 2009.

[40] B. W. Kooi, G. A. K. van Voorn, and K. P. Das, "Stabilization and complex dynamics in a predator-prey model with predator suffering from an infectious disease," Ecological Complexity, vol. 8, no. 1, pp. 113-122, 2011.

[41] M. Delgado, M. Molina-Becerra, and A. Suárez, "Analysis of an age-structured predator-prey model with disease in the prey," Nonlinear Analysis: Real World Applications, vol. 7, no. 4, pp. 853-871, 2006.

[42] R. M. Anderson and R. M. May, "The population dynamics of microparasites and their invertebrate hosts," Philosophical Transactions of the Royal Society B: Biological Sciences, vol. 291, no. 1054 , pp. 451-524, 1981.

[43] J. Chattopadhyay and O. Arino, "A predator-prey model with disease in the prey," Nonlinear Analysis: Theory, Methods \& Applications, vol. 36, pp. 747-766, 1999.

[44] R. M. Anderson and R. M. May, "The invasion, persistence and spread of infectious diseases within animal and plant communities.," Philosophical transactions of the Royal Society of London. Series B: Biological sciences, vol. 314, no. 1167, pp. 533570, 1986.

[45] Y. Xiao and L. Chen, "Modeling and analysis of a predator-prey model with disease in the prey," Mathematical Biosciences, vol. 171 , no. 1, pp. 59-82, 2001. 
[46] D. Greenhalgh and M. Haque, "A predator-prey model with disease in the prey species only," Mathematical Methods in the Applied Sciences, vol. 30, no. 8, pp. 911-929, 2007.

[47] S. Sinha, O. P. Misra, and J. Dhar, "Modelling a predator-prey system with infected prey in polluted environment," Applied Mathematical Modelling, vol. 34, no. 7, pp. 1861-1872, 2010.

[48] E. Venturino, "Mathematical population dynamics: analysis of heterogeneity, vol. one: theory of epidemics," in Epidemics in Predator-Prey Models: Disease among the Prey, pp. 33-50, Wuertz Publishing, Winnipeg, Canada, 1995.

[49] M. Liu, Z. Jin, and M. Haque, "An impulsive predator-prey model with communicable disease in the prey species only," Nonlinear Analysis: Real World Applications, vol. 10, no. 5, pp. 3098-3111, 2009.

[50] R. Bhattacharyya and B. Mukhopadhyay, "On an ecoepidemiological model with prey harvesting and predator switching: local and global perspectives," Nonlinear Analysis. Real World Applications, vol. 11, no. 5, pp. 3824-3833, 2010.

[51] E. Venturino, "Epidemics in predator-prey models: disease in the predators," IMA Journal of Mathematics Applied in Medicine and Biology, vol. 19, no. 3, pp. 185-205, 2002.

[52] Y. Xiao and L. Chen, "A ratio-dependent predator-prey model with disease in the prey," Applied Mathematics and Computation, vol. 131, no. 2-3, pp. 397-414, 2002.

[53] M. Haque and E. Venturino, "An ecoepidemiological model with disease in predator: the ratio-dependent case," Mathematical Methods in the Applied Sciences, vol. 30, no. 14, pp. 1791-1809, 2007.

[54] M. Haque, "A predator-prey model with disease in the predator species only," Nonlinear Analysis. Real World Applications, vol. 11, no. 4, pp. 2224-2236, 2010.

[55] F. M. Hilker and K. Schmitz, "Disease-induced stabilization of predator-prey oscillations," Journal of Theoretical Biology, vol. 255, no. 3, pp. 299-306, 2008.

[56] P. Auger, R. Mchich, T. Chowdhury, G. Sallet, M. Tchuente, and J. Chattopadhyay, "Effects of a disease affecting a predator on the dynamics of a predator-prey system," Journal of Theoretical Biology, vol. 258, no. 3, pp. 344-351, 2009.

[57] Y.-H. Hsieh and C.-K. Hsiao, "Predator-prey model with disease infection in both populations," Mathematical Medicine and Biology, vol. 25, no. 3, pp. 247-266, 2008.

[58] K. pada Das, K. Kundu, and J. Chattopadhyay, "A predatorprey mathematical model with both the populations affected by diseases," Ecological Complexity, vol. 8, no. 1, pp. 68-80, 2011.

[59] X. Gao, Q. Pan, M. He, and Y. Kang, "A predator-prey model with diseases in both prey and predator," Physica A. Statistical Mechanics and its Applications, vol. 392, no. 23, pp. 5898-5906, 2013.

[60] C. J. Briggs and M. F. Hoopes, "Stabilizing effects in spatial parasitoid-host and predator-prey models: a review," Theoretical Population Biology, vol. 65, no. 3, pp. 299-315, 2004.

[61] S. Chaudhuri, A. Costamagna, and E. Venturino, "Epidemics spreading in predator-prey systems," International Journal of Computer Mathematics, vol. 89, no. 4, pp. 561-584, 2012.

[62] H. W. Hethcote and J. W. van Ark, Modeling HIV Transmission and AIDS in the United States, Springer, New York, NY, USA, 1992.

[63] R. D. Holt and J. Pickering, "Infectious disease and species coexistence: a model of Lotka- Volterra form," American Naturalist, vol. 126, no. 2, pp. 196-211, 1985.
[64] E. Venturino, "On epidemics crossing the species barrier in interacting population models," Varahmihir Journal of Mathematical Sciences, vol. 6, no. 1, pp. 247-263, 2006. 


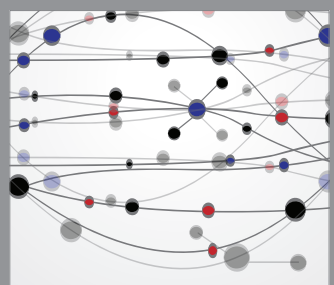

The Scientific World Journal
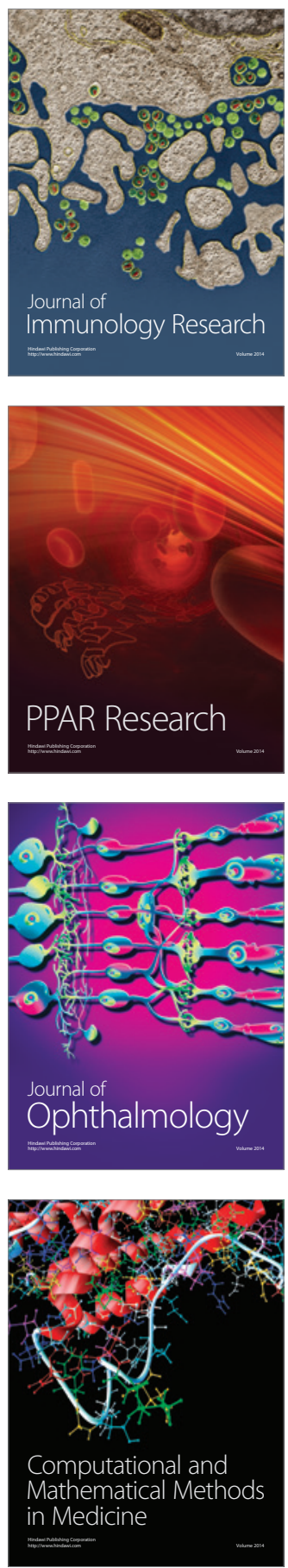

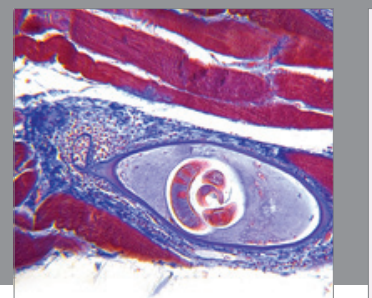

Gastroenterology

Research and Practice
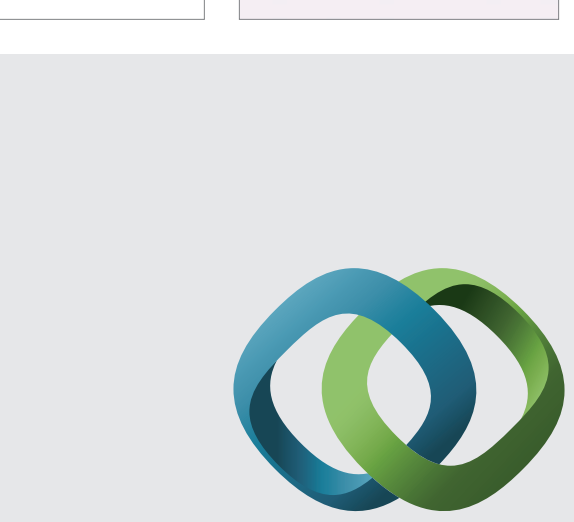

\section{Hindawi}

Submit your manuscripts at

http://www.hindawi.com
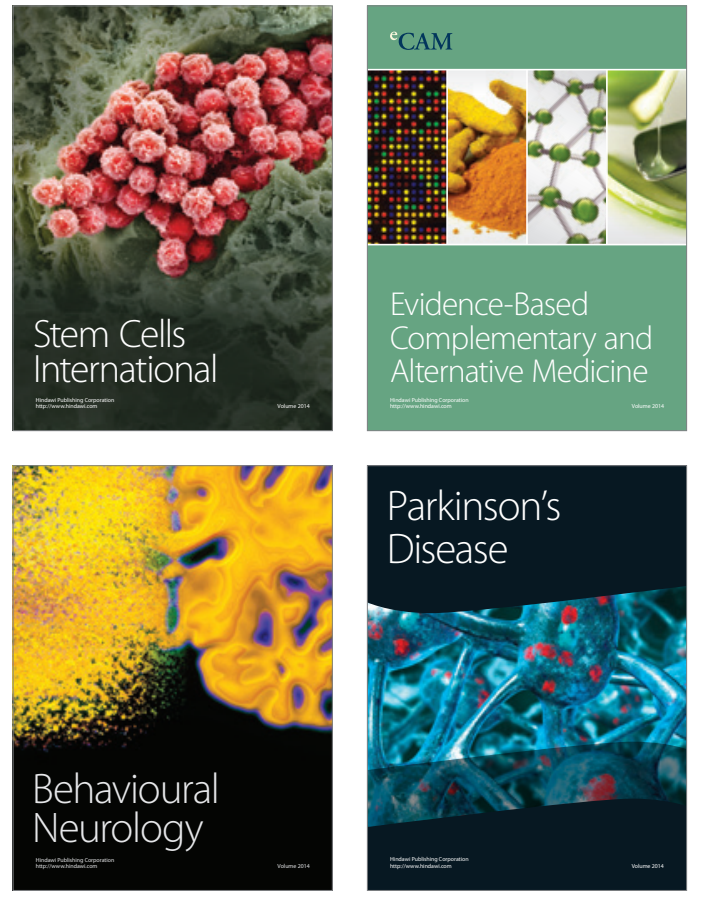
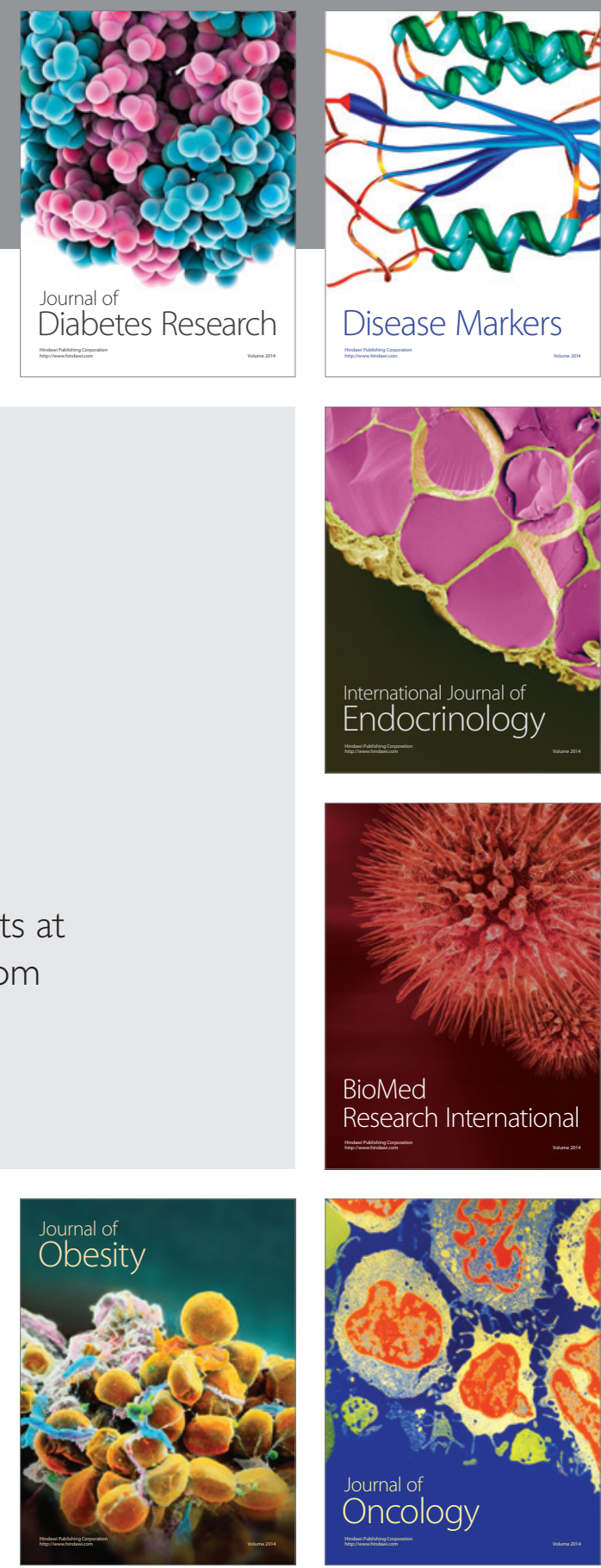

Disease Markers
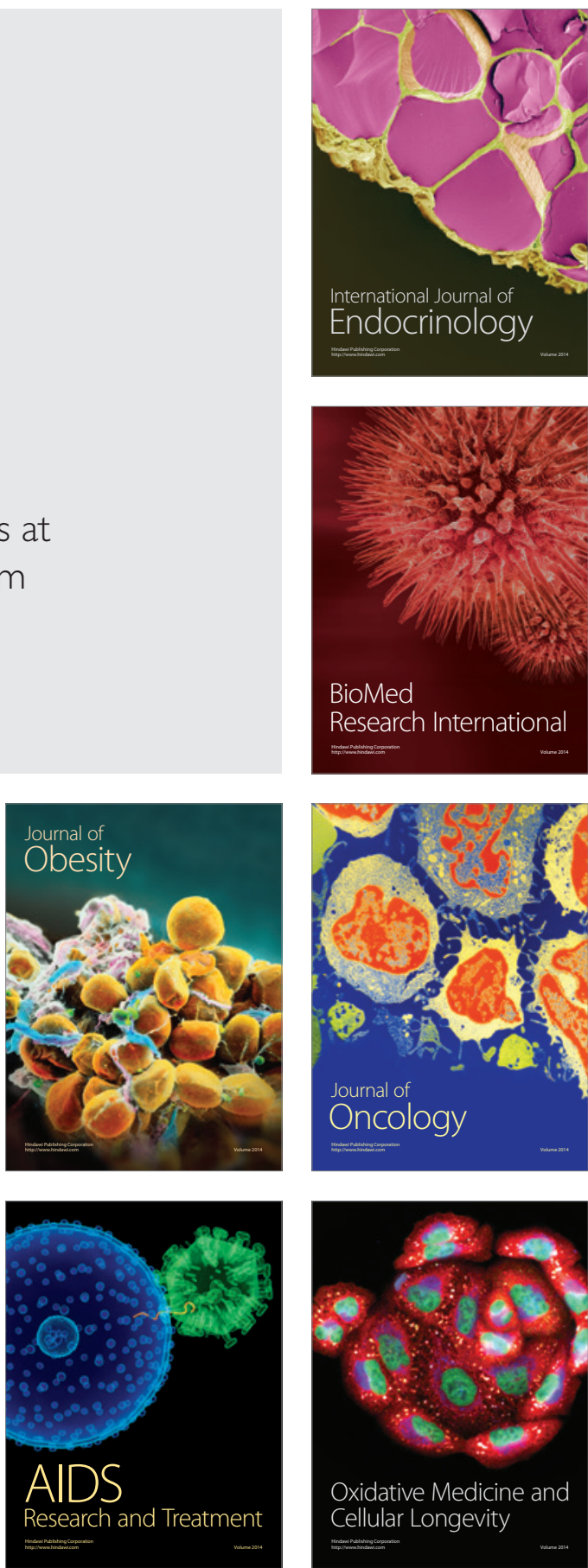